\title{
Existence and uniqueness of the solution to a coupled fractional diffusion system
}

\author{
Lang Li, Lingyu Jin and Shaomei Fang*
}

\section{"Correspondence:}

dz90@scau.edu.cn

Department of Applied

Mathematics, South China

Agricultural University, Guangzhou,

China

\begin{abstract}
In this paper, we consider the initial boundary value problem for a coupled fractional diffusion system. By using eigenfunction expansions and a priori estimates, we establish the existence and uniqueness of the weak solution and then the regularity of the solution.
\end{abstract}

Keywords: fractional diffusion system; initial boundary value problem; weak solution

\section{Introduction}

The traditional diffusion equation has commonly been used to describe the phenomenon of Brownian motion. However, it may not be adequate for the motion of the particles if they do not obey the law of Markov diffusion. An example is the diffusion that takes place in a highly heterogeneous aquifer, the probability density may have a heavier tail than the Gaussia density. This phenomenon, called anomalous diffusion, can be well modeled by fractional diffusion equations, including time-fractional diffusion equations, space fractional diffusion equations, and time-space fractional diffusion equations. The main reason is that the fractional derivative is nonlocal, which provides an excellent instrument to model the processes with fractal geometry, hereditary, and non-Markovian properties.

Recently, the fractional diffusion equation has attracted a great deal of interest and more applications of it have been investigated to represent many natural processes in physics chemistry, biology, medicine, food processing, aerodynamics, etc. [1-7]. By using different methods, many authors have discussed the mathematical properties of the solution to the fractional differential equation and its numerical result. For example, by using a finite sine transform technique, Agarwal [8] obtained the general solution to a fractional diffusion equation and presented some numerical results to show the influence of the fractional order $\alpha$ on the solution. Meanwhile, Mainardi solved a fractional diffusion equation by using the Laplace transform method [9]. In [10], Eidelman and Kochubei considered the Cauchy problem for fractional differential equations, they constructed the fundamental solution and proved the maximum principle. Furthermore, Luchko established the maximum principle for the generalized fractional differential equations [11]. Then Luchko extended the result to the generalized multi-term time-fractional diffusion equation [12]. In [13], Sakamoto and Yamamoto established the existence of the weak solution and asymptotic behavior for fractional diffusion-wave equation and then considered its application to some inverse problems.

(c) $2015 \mathrm{Li}$ et al. This article is distributed under the terms of the Creative Commons Attribution 4.0 International License (http://creativecommons.org/licenses/by/4.0/), which permits unrestricted use, distribution, and reproduction in any medium, provided you give appropriate credit to the original author(s) and the source, provide a link to the Creative Commons license, and indicate if changes were made. 
In this paper, we extend the existing result to a coupled system and consider the following coupled fractional diffusion equations

$$
\begin{aligned}
& \partial_{t}^{\alpha} u(x, t)=L u(x, t)+F_{1}(u, v), \\
& \partial_{t}^{\alpha} v(x, t)=L v(x, t)+F_{2}(u, v), \\
& u=v=0 \quad \text { on } x \in \partial \Omega, t \in(0, T], \\
& \left.u\right|_{t=0}=a_{1}(x),\left.\quad v\right|_{t=0}=a_{2}(x), \quad x \in \Omega,
\end{aligned}
$$

where $\Omega$ is a bounded domain in $\mathcal{R}^{d}$ with sufficiently smooth boundary $\partial \Omega, L$ is a symmetric uniformly elliptic operator, $F_{1}(u, v)$ and $F_{2}(u, v)$ are the forcing terms depending on $u$ and $v$, the initial data $a_{1}(x)$ and $a_{2}(x)$ are given functions on $\Omega$, and $T>0$ is a fixed value.

Here $\partial_{t}^{\alpha}$ is the Caputo fractional derivative of order $\alpha$ and is defined by

$$
\partial_{t}^{\alpha} g(t)=\frac{1}{\Gamma(n-\alpha)} \int_{0}^{t}(t-\tau)^{n-\alpha-1} \frac{d^{n}}{d \tau^{n}} g(\tau) d \tau, \quad n-1<\alpha<n, n \in \mathcal{N},
$$

where $\Gamma(\cdot)$ is the Gamma function.

Since we address fractional diffusion equations, we restrict ourselves to the two cases $0<\alpha<1$ and $1<\alpha<2$. If $0<\alpha<1$, this system describes a relaxation process (subdiffusion). If $1<\alpha<2$, an oscillation may occur (super-diffusion). If $\alpha=1$ and $L=\Delta$, it corresponds to a standard reaction-diffusion system. So we can regard the above system as the generalization of reaction-diffusion system to fractional order.

If $L=\Delta, F_{1}(u, v)=f(u)$, and $F_{2}(u, v)=g(v)$, this system is decoupled and becomes two fractional nonlinear diffusion equations. For the one nonlinear fractional diffusion equation of type (1) with unknown function, many authors have considered the initial boundary value problem. For example, Gafiychuk and Datsko investigated possible scenarios of pattern formations in fractional reaction-diffusion systems with initial boundary (Neumann) conditions [14]. In [15], Luchko established the existence and uniqueness of the solution for the initial boundary (Dirichlet) value problem. In this paper, motivated by the above discussion, we extend it to a more involved and complex system and study the existence of the solution.

The fractional diffusion system with Dirichlet boundary conditions arises in the heat transfer theory, population ecology, hydrodynamics, etc., and this problem has strong backgrounds in biology and mathematical physics. Among the application of such systems one can find the description of the evolution of the population densities of two competing species, one the natural species and the other a genetically engineered species [16]. Because of the use of genetically modified organisms, the ecosystems may be disrupted. So it is important to study the dynamics of two competing species. Moreover, due to the implementation of fractional derivatives, many new phenomena in such systems have been found. The analysis of such systems has attracted many authors' interests, both from the point view of qualitative analysis and computer simulations. For example, long time behavior of solutions of autonomous two-component incommensurate fractional dynamical systems was investigated [17]. In [18], the authors studied the self-organization phenomena in time-fractional reaction-diffusion systems with multiple homogeneous state. The simulation results were also presented for the case of an incommensurate time-fractional reaction-diffusion system with a cubic nonlinearity. 
To our knowledge, there have not been many papers published concerning the existence and uniqueness of the solution to equations (1)-(4). The goal of this paper is to prove the existence result as regards the weak solution and establish some results on the regularity of the solution.

\section{Preliminaries}

Let $L^{2}(\Omega), H^{1}(\Omega), H^{2}(\Omega)$ be the usual Sobolev spaces. $\dot{L}^{2}(\Omega)$ is a homogeneous Hilbert space defined as follows

$$
\dot{L}^{2}(\Omega)=\left\{u \in L^{2}(\Omega), u=0 \text { on } x \in \partial \Omega\right\} .
$$

Let $H=\dot{L}^{2}(\Omega) \times \dot{L}^{2}(\Omega)$ be the product Hilbert space with the norm

$$
\|\phi\|_{H}=\sqrt{\|u\|_{L^{2}}^{2}+\|v\|_{L^{2}}^{2}}
$$

for any $\phi=(u, v) \in H$.

Define $V=V_{1} \times V_{2}$, where

$$
\begin{aligned}
& V_{1}=\left\{u \in H^{2}(\Omega) \cap H_{0}^{1}(\Omega), u=0 \text { on } x \in \partial \Omega\right\}, \\
& V_{2}=\left\{v \in H^{2}(\Omega) \cap H_{0}^{1}(\Omega), v=0 \text { on } x \in \partial \Omega\right\} .
\end{aligned}
$$

Then the function space $V$ is a product Hilbert space with the induced norm

$$
\|\phi\|_{V}=\sqrt{\|u\|_{H^{2}}^{2}+\|v\|_{H^{2}}^{2}}
$$

where $\phi=(u, v) \in V$.

Let $V^{\prime}$ be the dual space of $V$, we have

$$
V \hookrightarrow H=H^{\prime} \hookrightarrow V^{\prime}
$$

where the embeddings above are dense and continuous. We denote by $\langle\phi, \psi\rangle$ the duality between $\phi \in V$ and $\psi \in V^{\prime}$.

Under the assumption that $L$ is an uniformly elliptic operator and $\mathcal{D}(-L)=H^{2}(\Omega) \cap$ $H_{0}^{1}(\Omega)$, we define

$$
-L \varphi_{n}=\lambda_{n} \varphi_{n}, \quad n=1,2, \ldots,
$$

where $\left\{\lambda_{n}\right\}_{n=1}^{\infty}$ is the eigenvalues of $-L$ and $\left\{\varphi_{n}\right\}_{n=1}^{\infty}$ is the corresponding orthonormal eigenfunctions which belong to $H^{2}(\Omega) \cap H_{0}^{1}(\Omega)$. It follows that the sequence $\left\{\varphi_{n}\right\}_{n=1}^{\infty}$ is an orthonormal basis in $L^{2}(\Omega)$. For any $n \in \mathcal{N}, \lambda_{n}>0$. Without loss of generality, we set

$$
0<\lambda_{1} \leq \lambda_{2} \leq \cdots
$$

Then we define

$$
\mathcal{D}\left((-L)^{\gamma}\right)=\left\{\psi \in L^{2}(\Omega) ; \sum_{n=1}^{\infty} \lambda_{n}^{2 \gamma}\left|\left(\psi, \varphi_{n}\right)\right|^{2}<\infty\right\},
$$


for any $\gamma \in \mathcal{R}$. It follows that $\mathcal{D}\left((-L)^{\gamma}\right)$ is a Hilbert space with the norm

$$
\|\psi\|_{\mathcal{D}\left((-L)^{\gamma}\right)}=\left\{\sum_{n=1}^{\infty} \lambda_{n}^{2 \gamma}\left|\left(\psi, \varphi_{n}\right)\right|^{2}\right\}^{\frac{1}{2}} .
$$

Since $\mathcal{D}\left((-L)^{\gamma}\right) \subset L^{2}(\Omega)$ for $\gamma>0$ and $\left(L^{2}(\Omega)\right)^{\prime}=L^{2}(\Omega)$, we have

$$
\mathcal{D}\left((-L)^{\gamma}\right) \subset L^{2}(\Omega)=\left(L^{2}(\Omega)\right)^{\prime} \subset\left(\mathcal{D}\left((-L)^{\gamma}\right)\right)^{\prime}
$$

Set $\left(\mathcal{D}\left((-L)^{\gamma}\right)\right)^{\prime}=\mathcal{D}\left((-L)^{-\gamma}\right)$. Then it is easy to show that $\mathcal{D}\left((-L)^{-\gamma}\right)$ is a Hilbert space with the norm

$$
\|f\|_{\mathcal{D}\left((-L)^{-\gamma}\right)}=\left\{\left.\left.\sum_{n=1}^{\infty} \lambda_{n}^{-2 \gamma}\right|_{-\gamma}\left\langle f, \varphi_{n}\right\rangle_{\gamma}\right|^{2}\right\}^{\frac{1}{2}} .
$$

According to Riesz representation theorem, we have

$$
{ }_{-\gamma}\left\langle f, \varphi_{n}\right\rangle_{\gamma}=\left(f, \varphi_{n}\right)
$$

if $f \in L^{2}(\Omega)$ and $\varphi_{n} \in \mathcal{D}\left((-L)^{\gamma}\right)$.

The forcing terms are assumed to satisfy the following assumptions:

(A.1)

$$
\begin{aligned}
& \left\|F_{1}(u, v)\right\|_{L^{2}(\Omega)} \leq K_{1}\left(1+\|u\|_{L^{2}(\Omega)}+\|v\|_{L^{2}(\Omega)}\right), \\
& \left\|F_{2}(u, v)\right\|_{L^{2}(\Omega)} \leq K_{2}\left(1+\|u\|_{L^{2}(\Omega)}+\|v\|_{L^{2}(\Omega)}\right),
\end{aligned}
$$

$$
\begin{aligned}
& \left\|F_{1}(u, v)\right\|_{H^{2}(\Omega)} \leq K_{3}\left(1+\|u\|_{H^{2}(\Omega)}+\|v\|_{H^{2}(\Omega)}\right), \\
& \left\|F_{2}(u, v)\right\|_{H^{2}(\Omega)} \leq K_{4}\left(1+\|u\|_{H^{2}(\Omega)}+\|v\|_{H^{2}(\Omega)}\right),
\end{aligned}
$$

$$
\begin{aligned}
& \left\|F_{1}(u, v)-F_{1}\left(u^{\prime}, v^{\prime}\right)\right\|_{L^{2}(\Omega)} \leq L_{1}\left(\left\|u-u^{\prime}\right\|_{L^{2}(\Omega)}+\left\|v-v^{\prime}\right\|_{L^{2}(\Omega)}\right) \\
& \left\|F_{2}(u, v)-F_{2}\left(u^{\prime}, v^{\prime}\right)\right\|_{L^{2}(\Omega)} \leq L_{2}\left(\left\|u-u^{\prime}\right\|_{L^{2}(\Omega)}+\left\|v-v^{\prime}\right\|_{L^{2}(\Omega)}\right)
\end{aligned}
$$

for any $t \in[0, T],(u, v) \in H,\left(u^{\prime}, v^{\prime}\right) \in H$.

Now we give some examples to show the assumptions (A.1)-(A.3) can be satisfied.

Example 1 Suppose that $F_{1}(u, v)=u-v$ and $F_{2}(u, v)=u-v+\mathcal{A}$, where $\mathcal{A}$ is an external parameter, it is easy to show the assumptions (A.1)-(A.3) are satisfied.

Example 2 Suppose that $F_{1}(u, v)=u-g\left(|u|^{2}\right) u-v$ and $F_{2}(u, v)=v-u$, where $g(r)=0$, if $r>N ; g(r)=r-N$, if $r \leq N$ and $0 \leq g^{\prime}(r) \leq C, 0 \leq g^{\prime \prime}(r) \leq C$. We can also verify $F_{1}$ and $F_{2}$ satisfy the assumptions; for more details, see pp.583 of [19].

When no confusion arises, we simply denote $L^{p}(\Omega)$ by $L^{p}$ and $H^{p}(\Omega)$ by $H^{p}$ for $1 \leq p \leq \infty$. We denote by $C$ a positive constant which may change from one line to the next line. 
Moreover, the Mittag-Leffler function is defined as follows

$$
E_{\alpha, \beta}(z):=\sum_{k=0}^{\infty} \frac{z^{k}}{\Gamma(\alpha k+\beta)}, \quad z \in \mathcal{C},
$$

where $\alpha>0$ and $\beta \in \mathcal{R}$ are arbitrary constants.

Now, we give three lemmas concerning the Mittag-Leffler function which will be used later.

Lemma 1 [2] Let $0<\alpha<2$ and $\beta \in \mathbb{R}$ be arbitrary. We suppose that $\mu$ is such that $\pi \alpha / 2<$ $\mu<\min \{\pi, \pi \alpha\}$. Then there exists a constant $C_{1}=C_{1}(\alpha, \beta, \mu)>0$ such that

$$
\left|E_{\alpha, \beta}(z)\right| \leq \frac{C_{1}}{1+|z|}, \quad \mu \leq|\arg (z)| \leq \pi .
$$

Lemma 2 [13] For $\lambda>0, \alpha>0$, and positive integer $m \in \mathbb{N}$, we have

$$
\frac{d^{m}}{d t^{m}} E_{\alpha, 1}\left(-\lambda t^{\alpha}\right)=-\lambda t^{\alpha-m} E_{\alpha, \alpha-m+1}\left(-\lambda t^{\alpha}\right), \quad t>0,
$$

and

$$
\frac{d}{d t}\left(t^{\alpha-1} E_{\alpha, \alpha}\left(-\lambda t^{\alpha}\right)\right)=t^{\alpha-2} E_{\alpha, \alpha-1}\left(-\lambda t^{\alpha}\right), \quad t \geq 0 .
$$

Lemma 3 [20] For $0<\alpha<1$, we have

$$
E_{\alpha, \alpha}(-x) \geq 0, \quad x \geq 0
$$

\section{Existence result}

In this paper, we are interested in studying the existence of weak solutions. So we first give the definition of weak solutions as follows.

Definition $(u, v)$ is called a weak solution to equations (1)-(4) if equations (1)-(2) hold in $H$ and $(u, v) \in V$ for almost all $t \in(0, T)$. Moreover, $(u, v) \in C\left([0, T] ; \mathcal{D}\left((-L)^{-\gamma}\right)\right) \times$ $C\left([0, T] ; \mathcal{D}\left((-L)^{-\gamma}\right)\right)$ and satisfy

$$
\lim _{t \rightarrow 0}\left\|u(\cdot, t)-a_{1}\right\|_{\mathcal{D}\left((-L)^{-\gamma}\right)}=\lim _{t \rightarrow 0}\left\|v(\cdot, t)-a_{2}\right\|_{\mathcal{D}\left((-L)^{-\gamma}\right)}=0
$$

with some $\gamma>0$.

Now we state our first main result as follows.

Theorem 1 (i) Let $\frac{1}{2} \leq \alpha<1$, and the initial data $\left(a_{1}, a_{2}\right) \in H$. Then there exists a unique weak solution $(u, v)$ to equations (1)-(4) with

$$
(u, v) \in C((0, T] ; V) \cap C([0, T] ; H), \quad\left(\partial_{t}^{\alpha} u, \partial_{t}^{\alpha} v\right) \in C((0, T] ; H),
$$

and the following inequalities hold

$$
\|u\|_{L^{2}}+\|v\|_{L^{2}} \leq C, \quad t \in[0, T],
$$




$$
\begin{aligned}
& \|u\|_{H^{2}}+\|v\|_{H^{2}} \leq C, \quad t \in(0, T] \\
& \left\|\partial_{t}^{\alpha} u\right\|_{L^{2}}+\left\|\partial_{t}^{\alpha} v\right\|_{L^{2}} \leq C, \quad t \in(0, T] .
\end{aligned}
$$

(ii) Let $\frac{1}{2} \leq \alpha<1$, and the initial data $\left(a_{1}, a_{2}\right) \in V$. Then there exists a unique solution $(u, v)$ to equations (1)-(4) with

$$
(u, v) \in C([0, T] ; V), \quad\left(\partial_{t}^{\alpha} u, \partial_{t}^{\alpha} v\right) \in C([0, T] ; H),
$$

and the following inequalities hold

$$
\begin{aligned}
& \|u\|_{H^{2}}+\|v\|_{H^{2}} \leq C, \quad t \in[0, T], \\
& \left\|\partial_{t}^{\alpha} u\right\|_{L^{2}}+\left\|\partial_{t}^{\alpha} v\right\|_{L^{2}} \leq C, \quad t \in[0, T] .
\end{aligned}
$$

Proof (i) Using the Fourier method, we have eigenfunction expansions of the solution to equations (1)-(4) (p.230 of [1]),

$$
\begin{aligned}
u(x, t)= & \sum_{n=1}^{\infty}\left(a_{1}, \varphi_{n}\right) E_{\alpha, 1}\left(-\lambda_{n} t^{\alpha}\right) \varphi_{n}(x) \\
& +\sum_{n=1}^{\infty}\left(\int_{0}^{t}\left(F_{1}(u, v, \tau), \varphi_{n}\right)(t-\tau)^{\alpha-1} E_{\alpha, \alpha}\left(-\lambda_{n}(t-\tau)^{\alpha}\right) d \tau\right) \varphi_{n}(x), \\
v(x, t)= & \sum_{n=1}^{\infty}\left(a_{2}, \varphi_{n}\right) E_{\alpha, 1}\left(-\lambda_{n} t^{\alpha}\right) \varphi_{n}(x) \\
& +\sum_{n=1}^{\infty}\left(\int_{0}^{t}\left(F_{2}(u, v, \tau), \varphi_{n}\right)(t-\tau)^{\alpha-1} E_{\alpha, \alpha}\left(-\lambda_{n}(t-\tau)^{\alpha}\right) d \tau\right) \varphi_{n}(x) .
\end{aligned}
$$

Thanks to Lemma 1, Hölder's inequality and the assumption (A.1), we can deduce

$$
\begin{aligned}
\|u(\cdot, t)\|_{L^{2}}^{2} \leq & \sum_{n=1}^{\infty}\left|\left(a_{1}, \varphi_{n}\right) E_{\alpha, 1}\left(-\lambda_{n} t^{\alpha}\right)\right|^{2} \\
& +\sum_{n=1}^{\infty}\left|\left(\int_{0}^{t}\left(F_{1}(u, v, \tau), \varphi_{n}\right)(t-\tau)^{\alpha-1} E_{\alpha, \alpha}\left(-\lambda_{n}(t-\tau)^{\alpha}\right) d \tau\right)\right|^{2} \\
\leq & \sum_{n=1}^{\infty}\left|\left(a_{1}, \varphi_{n}\right)\right|^{2}\left(\frac{1}{1+\lambda_{n} t^{\alpha}}\right)^{2} \\
& +\sum_{n=1}^{\infty} \int_{0}^{t}\left|\left(F_{1}(u, v, \tau), \varphi_{n}\right)\right|^{2} d \tau \int_{0}^{t} \tau^{2 \alpha-2}\left|E_{\alpha, \alpha}\left(-\lambda_{n} \tau^{\alpha}\right)\right|^{2} d \tau \\
\leq & \sum_{n=1}^{\infty}\left|\left(a_{1}, \varphi_{n}\right)\right|^{2}\left(\frac{1}{1+\lambda_{n} t^{\alpha}}\right)^{2} \\
& +\sum_{n=1}^{\infty} \int_{0}^{t}\left|\left(F_{1}(u, v, \tau), \varphi_{n}\right)\right|^{2} d \tau \int_{0}^{t} \tau^{2 \alpha-2}\left(\frac{1}{1+\lambda_{n} \tau^{\alpha}}\right)^{2} d \tau \\
\leq & \left\|a_{1}\right\|_{L^{2}}^{2}+T^{2 \alpha-1} \int_{0}^{t}\left\|F_{1}(u, v, \tau)\right\|_{L^{2}}^{2} d \tau \\
\leq & \left\|a_{1}\right\|_{L^{2}}^{2}+K_{1} T^{2 \alpha}+K_{1} T^{2 \alpha-1} \int_{0}^{t}\left(\|u\|_{L^{2}}^{2}+\|v\|_{L^{2}}^{2}\right) d \tau .
\end{aligned}
$$


Similarly, we can also obtain

$$
\|v(\cdot, t)\|_{L^{2}}^{2} \leq\left\|a_{2}\right\|_{L^{2}}^{2}+K_{2} T^{2 \alpha}+K_{2} T^{2 \alpha-1} \int_{0}^{t}\left(\|u\|_{L^{2}}^{2}+\|v\|_{L^{2}}^{2}\right) d \tau,
$$

together with equation (7), we arrive at

$$
\|u(\cdot, t)\|_{L^{2}}^{2}+\|v(\cdot, t)\|_{L^{2}}^{2} \leq C\left(\left\|a_{1}\right\|_{L^{2}}^{2},\left\|a_{2}\right\|_{L^{2}}^{2}, K_{1}, K_{2}, T, \alpha\right)+C \int_{0}^{t}\left(\|u\|_{L^{2}}^{2}+\|v\|_{L^{2}}^{2}\right) d \tau,
$$

where we used the fact $\frac{1}{2} \leq \alpha<1$. By using Gronwall's inequality, we have

$$
\|u(\cdot, t)\|_{L^{2}}^{2}+\|v(\cdot, t)\|_{L^{2}}^{2} \leq C\left(\left\|a_{1}\right\|_{L^{2}}^{2},\left\|a_{2}\right\|_{L^{2}}^{2}, K_{1}, K_{2}, T, \alpha\right) .
$$

Under the assumption (A.1), it follows that

$$
\left\|F_{1}(u, v, t)\right\|_{L^{\infty}\left(0, T ; L^{2}\right)} \leq C, \quad\left\|F_{2}(u, v, t)\right\|_{L^{\infty}\left(0, T ; L^{2}\right)} \leq C .
$$

From equation (7), it is easy to see that $u \in C\left([0, T] ; L^{2}\right)$ for $\sum_{n=1}^{\infty}\left(a_{1}, \varphi_{n}\right) E_{\alpha, 1}\left(-\lambda_{n} t^{\alpha}\right) \varphi_{n}(x)$ and $\sum_{n=1}^{\infty}\left(\int_{0}^{t}\left(F_{1}(u, v, \tau), \varphi_{n}\right)(t-\tau)^{\alpha-1} E_{\alpha, \alpha}\left(-\lambda_{n}(t-\tau)^{\alpha}\right) d \tau\right) \varphi_{n}(x)$ are convergent in $L^{2}$ uniformly in $t \in[0, T]$. Similarly, we have $v \in C\left([0, T] ; L^{2}\right)$ from equation (8).

From equation (5) and equation (6), we then obtain

$$
\begin{aligned}
\partial_{t}^{\alpha} u(x, t)= & -\sum_{n=1}^{\infty} \lambda_{n}\left(a_{1}, \varphi_{n}\right) E_{\alpha, 1}\left(-\lambda_{n} t^{\alpha}\right) \varphi_{n}(x)+\sum_{n=1}^{\infty}\left(F_{1}(u, v, t), \varphi_{n}\right) \varphi_{n}(x) \\
& -\sum_{n=1}^{\infty} \lambda_{n}\left(\int_{0}^{t}\left(F_{1}(u, v, \tau), \varphi_{n}\right)(t-\tau)^{\alpha-1} E_{\alpha, \alpha}\left(-\lambda_{n}(t-\tau)^{\alpha}\right) d \tau\right) \varphi_{n}(x), \\
\partial_{t}^{\alpha} v(x, t)= & -\sum_{n=1}^{\infty} \lambda_{n}\left(a_{2}, \varphi_{n}\right) E_{\alpha, 1}\left(-\lambda_{n} t^{\alpha}\right) \varphi_{n}(x)+\sum_{n=1}^{\infty}\left(F_{2}(u, v, t), \varphi_{n}\right) \varphi_{n}(x) \\
& -\sum_{n=1}^{\infty} \lambda_{n}\left(\int_{0}^{t}\left(F_{2}(u, v, \tau), \varphi_{n}\right)(t-\tau)^{\alpha-1} E_{\alpha, \alpha}\left(-\lambda_{n}(t-\tau)^{\alpha}\right) d \tau\right) \varphi_{n}(x) .
\end{aligned}
$$

Therefore, by Lemma 1, Lemma 2 and Lemma 3, we have

$$
\begin{aligned}
\left\|\partial_{t}^{\alpha} u(\cdot, t)\right\|_{L^{2}}^{2} \leq & \sum_{n=1}^{\infty} \lambda_{n}^{2}\left|\left(a_{1}, \varphi_{n}\right)\right|^{2}\left|E_{\alpha, 1}\left(-\lambda_{n} t^{\alpha}\right)\right|^{2}+\sum_{n=1}^{\infty}\left|\left(F_{1}(u, v, t), \varphi_{n}\right)\right|^{2} \\
& +\sum_{n=1}^{\infty} \lambda_{n}^{2}\left|\int_{0}^{t}\left(F_{1}(u, v, \tau), \varphi_{n}\right)(t-\tau)^{\alpha-1} E_{\alpha, \alpha}\left(-\lambda_{n}(t-\tau)^{\alpha}\right) d \tau\right|^{2} \\
\leq & \sum_{n=1}^{\infty} \lambda_{n}^{2}\left|\left(a_{1}, \varphi_{n}\right)\right|^{2}\left(\frac{1}{1+\lambda_{n} t^{\alpha}}\right)^{2}+\sum_{n=1}^{\infty}\left|\left(F_{1}(u, v, t), \varphi_{n}\right)\right|^{2} \\
& +\sum_{n=1}^{\infty} \lambda_{n}^{2} \sup _{0 \leq \tau \leq T}\left|\left(F_{1}(u, v, \tau), \varphi_{n}\right)\right|^{2}\left(\int_{0}^{t} \tau^{\alpha-1} E_{\alpha, \alpha}\left(-\lambda_{n} \tau^{\alpha}\right) d \tau\right)^{2} \\
\leq & \left\|a_{1}\right\|_{L^{2}}^{2} t^{-2 \alpha}+\left\|F_{1}(u, v)\right\|_{L^{2}}^{2}
\end{aligned}
$$




$$
\begin{aligned}
& +\sum_{n=1}^{\infty} \sup _{0 \leq \tau \leq T}\left|\left(F_{1}(u, v, \tau), \varphi_{n}\right)\right|^{2}\left(-\int_{0}^{t} \frac{d}{d \tau} E_{\alpha, 1}\left(-\lambda_{n} \tau^{\alpha}\right) d \tau\right)^{2} \\
\leq & \left\|a_{1}\right\|_{L^{2}}^{2} t^{-2 \alpha}+\left\|F_{1}(u, v)\right\|_{L^{2}}^{2} \\
& +\left\|F_{1}(u, v, t)\right\|_{L^{\infty}\left(0, T ; L^{2}\right)}^{2}\left(1-E_{\alpha, 1}\left(-\lambda_{n} t^{\alpha}\right)\right)^{2} \\
\leq & \left\|a_{1}\right\|_{L^{2}}^{2} t^{-2 \alpha}+C\left(K_{1}, K_{2}, T, \alpha,\left\|a_{1}\right\|_{L^{2}}^{2},\left\|a_{2}\right\|_{L^{2}}^{2}\right), \quad t \in(0, T],
\end{aligned}
$$

where we have used the fact

$$
\int_{0}^{t}\left|\tau^{\alpha-1} E_{\alpha, \alpha}\left(-\lambda_{n} \tau^{\alpha}\right)\right| d \tau=\int_{0}^{t} \tau^{\alpha-1} E_{\alpha, \alpha}\left(-\lambda_{n} \tau^{\alpha}\right) d \tau=-\frac{1}{\lambda_{n}} \int_{0}^{t} \frac{d}{d \tau} E_{\alpha, 1}\left(-\lambda_{n} \tau^{\alpha}\right) d \tau .
$$

Similarly, we can also get

$$
\left\|\partial_{t}^{\alpha} v(\cdot, t)\right\|_{L^{2}}^{2} \leq\left\|a_{2}\right\|_{L^{2}}^{2} t^{-2 \alpha}+C\left(K_{1}, K_{2}, T, \alpha,\left\|a_{1}\right\|_{L^{2}}^{2},\left\|a_{2}\right\|_{L^{2}}^{2}\right), \quad t \in(0, T] .
$$

From equations (1)-(2), we also know that

$$
\begin{aligned}
\|u(\cdot, t)\|_{H^{2}}+\|v(\cdot, t)\|_{H^{2}} \leq & C\left(\|L u(\cdot, t)\|_{L^{2}}+\|L v(\cdot, t)\|_{L^{2}}\right) \\
\leq & C\left(\left\|\partial_{t}^{\alpha} u(\cdot, t)\right\|_{L^{2}}+\left\|\partial_{t}^{\alpha} v(\cdot, t)\right\|_{L^{2}}+\left\|F_{1}(u, v)\right\|_{L^{2}}\right. \\
& \left.+\left\|F_{2}(u, v)\right\|_{L^{2}}\right) \\
\leq & C t^{-2 \alpha}+C, \quad t \in(0, T],
\end{aligned}
$$

where $C$ depends on $\left\|a_{1}\right\|_{L^{2}},\left\|a_{2}\right\|_{L^{2}}, T, K_{1}, K_{2}, \alpha$.

Owing to $\sum_{n=1}^{\infty} \lambda_{n}\left(a_{1}, \varphi_{n}\right) E_{\alpha, 1}\left(-\lambda_{n} t^{\alpha}\right) \varphi_{n}(x)$ and $\sum_{n=1}^{\infty} \lambda_{n}\left(a_{2}, \varphi_{n}\right) E_{\alpha, 1}\left(-\lambda_{n} t^{\alpha}\right) \varphi_{n}(x)$ being convergent in $t \in[\delta, T]$ with any given $\delta>0, \sum_{n=1}^{\infty}\left(F_{1}(u, v, t), \varphi_{n}\right) \varphi_{n}(x), \sum_{n=1}^{\infty}\left(F_{2}(u, v, t)\right.$, $\left.\varphi_{n}\right) \varphi_{n}(x), \sum_{n=1}^{\infty} \lambda_{n}\left(\int_{0}^{t}\left(F_{1}(u, v, \tau), \varphi_{n}\right)(t-\tau)^{\alpha-1} E_{\alpha, \alpha}\left(-\lambda_{n}(t-\tau)^{\alpha}\right) d \tau\right) \varphi_{n}(x)$, and $\sum_{n=1}^{\infty} \lambda_{n} \times$ $\left(\int_{0}^{t}\left(F_{2}(u, v, \tau), \varphi_{n}\right)(t-\tau)^{\alpha-1} E_{\alpha, \alpha}\left(-\lambda_{n}(t-\tau)^{\alpha}\right) d \tau\right) \varphi_{n}(x)$ being convergent in $t \in[0, T]$, we see that $\left(\partial_{t}^{\alpha} u, \partial_{t}^{\alpha} v\right) \in C((0, T] ; H)$ and $(u, v) \in C((0, T] ; V)$.

Next we prove the uniqueness.

Taking the duality pairing ${ }_{-\gamma}\langle\cdot, \cdot\rangle_{\gamma}$ of equations (1)-(4) with $\varphi_{n}$ and setting $u_{n}(t)=$ ${ }_{-\gamma}\left\langle u(x, t), \varphi_{n}\right\rangle_{\gamma}, v_{n}(t)={ }_{-\gamma}\left\langle v(x, t), \varphi_{n}\right\rangle_{\gamma}$, we obtain

$$
\begin{aligned}
& \partial_{t}^{\alpha} u_{n}(t)=-\lambda_{n} u_{n}(t)+F_{1 n}(t), \\
& \partial_{t}^{\alpha} v_{n}(t)=-\lambda_{n} v_{n}(t)+F_{2 n}(t), \\
& u_{n}(t)=v_{n}(t)=0, \quad t \in(0, T], \\
& \left.u_{n}\right|_{t=0}=\left(a_{1}, \varphi_{n}\right),\left.\quad v_{n}\right|_{t=0}=\left(a_{2}, \varphi_{n}\right) .
\end{aligned}
$$

Due to the existence of the ordinary fractional differential equations and the Lipschitz assumption (A.3), we obtain the existence and uniqueness of the solution to equations (11)-(14). Since $\left\{\varphi_{n}\right\}_{n=1}^{\infty}$ is a complete orthonormal basis in $L^{2}$, we get the uniqueness of the weak solution $(u, v)$ to equations (1)-(4).

Finally, we have to prove

$$
\lim _{t \rightarrow 0}\left\|u(\cdot, t)-a_{1}\right\|_{L^{2}}=0, \quad \lim _{t \rightarrow 0}\left\|v(\cdot, t)-a_{2}\right\|_{L^{2}}=0 .
$$


In fact,

$$
\begin{aligned}
\left\|u(\cdot, t)-a_{1}\right\|_{L^{2}}^{2}= & \sum_{n=1}^{\infty}\left|\left(a_{1}, \varphi_{n}\right)\left(E_{\alpha, 1}\left(-\lambda_{n} t^{\alpha}\right)-1\right)\right|^{2} \\
& +\sum_{n=1}^{\infty}\left|\int_{0}^{t}\left(F_{1}(u, v, \tau), \varphi_{n}\right)(t-\tau)^{\alpha-1} E_{\alpha, \alpha}\left(-\lambda_{n}(t-\tau)^{\alpha}\right) d \tau\right|^{2} \\
= & S_{1}(t)+S_{2}(t) .
\end{aligned}
$$

Obviously, $\lim _{t \rightarrow 0} S_{2}(t)=0$. Since

$$
S_{1}(t)=\sum_{n=1}^{\infty}\left|\left(a_{1}, \varphi_{n}\right)\left(E_{\alpha, 1}\left(-\lambda_{n} t^{\alpha}\right)-1\right)\right|^{2} \leq 2 \sum_{n=1}^{\infty}\left|\left(a_{1}, \varphi_{n}\right)\right|^{2}\left(\left(\frac{C}{1+\lambda_{n} t^{\alpha}}\right)^{2}+1\right)<\infty
$$

and

$$
\lim _{t \rightarrow 0}\left(E_{\alpha, 1}\left(-\lambda_{n} t^{\alpha}\right)-1\right)=0,
$$

by using the Lebesgue dominated convergence theorem, we have

$$
\lim _{t \rightarrow 0} S_{1}(t)=0 .
$$

Hence

$$
\lim _{t \rightarrow 0}\left\|u(\cdot, t)-a_{1}\right\|_{L^{2}}=0 .
$$

Similarly,

$$
\lim _{t \rightarrow 0}\left\|v(\cdot, t)-a_{2}\right\|_{L^{2}}=0 .
$$

Thus we complete the proof of (i).

(ii) Under the assumption that $\left(a_{1}, a_{2}\right) \in V$, we have

$$
\begin{aligned}
\|u(\cdot, t)\|_{H^{2}}^{2} & \\
\leq & C\|L u\|_{L^{2}}^{2} \\
\leq & C \sum_{n=1}^{\infty}\left|\lambda_{n}\left(a_{1}, \varphi_{n}\right) E_{\alpha, 1}\left(-\lambda_{n} t^{\alpha}\right)\right|^{2} \\
& +C \sum_{n=1}^{\infty}\left|\int_{0}^{t}\left(F_{1}(u, v, \tau), \varphi_{n}\right) \lambda_{n}(t-\tau)^{\alpha-1} E_{\alpha, \alpha}\left(-\lambda_{n}(t-\tau)^{\alpha}\right) d \tau\right|^{2} \\
\leq & C\left\|a_{1}\right\|_{H^{2}}^{2}+C \sum_{n=1}^{\infty} \sup _{0 \leq \tau \leq T}\left|\left(F_{1}(u, v, \tau), \varphi_{n}\right)\right|^{2}\left|\int_{0}^{t} \lambda_{n}(t-\tau)^{\alpha-1} E_{\alpha, \alpha}\left(-\lambda_{n}(t-\tau)^{\alpha}\right) d \tau\right|^{2} \\
\leq & C\left\|a_{1}\right\|_{H^{2}}^{2}+C \sum_{n=1}^{\infty} \sup _{0 \leq \tau \leq T}\left|\left(F_{1}(u, v, \tau), \varphi_{n}\right)\right|^{2}\left|\int_{0}^{t} \frac{d}{d \tau} E_{\alpha, 1}\left(-\lambda_{n} \tau^{\alpha}\right) d \tau\right|^{2}
\end{aligned}
$$




$$
\begin{aligned}
& \leq C\left\|a_{1}\right\|_{H^{2}}^{2}+C\left\|F_{1}(u, v)\right\|_{L^{\infty}\left(0, T ; L^{2}\right)}^{2} \\
& \leq C\left(\left\|a_{1}\right\|_{H^{2}}, T, K_{1}, K_{2}, \alpha\right), \quad t \in[0, T] .
\end{aligned}
$$

Similarly,

$$
\|v(\cdot, t)\|_{H^{2}}^{2} \leq C\left(\left\|a_{2}\right\|_{H^{2}}, T, K_{1}, K_{2}, \alpha\right), \quad t \in[0, T] .
$$

From equations (1)-(2), it follows that

$$
\left\|\partial_{t}^{\alpha} u(\cdot, t)\right\|_{L^{2}}+\left\|\partial_{t}^{\alpha} v(\cdot, t)\right\|_{L^{2}} \leq C\left(\left\|a_{1}\right\|_{H^{2}},\left\|a_{2}\right\|_{H^{2}}, T, K_{1}, K_{2}, \alpha\right), \quad t \in[0, T] .
$$

Next we have to prove

$$
\lim _{t \rightarrow 0}\left\|u(\cdot, t)-a_{1}\right\|_{\mathcal{D}\left((-L)^{-\gamma}\right)}^{2}=0, \quad \lim _{t \rightarrow 0}\left\|v(\cdot, t)-a_{2}\right\|_{\mathcal{D}\left((-L)^{-\gamma}\right)}^{2}=0 .
$$

In fact,

$$
\begin{aligned}
& \left\|u(\cdot, t)-a_{1}\right\|_{\mathcal{D}\left((-L)^{-\gamma}\right)}^{2} \\
& =\sum_{n=1}^{\infty} \frac{1}{\lambda_{n}^{2 \gamma}}\left|\left(a_{1}, \varphi_{n}\right) E_{\alpha, 1}\left(-\lambda_{n} t^{\alpha}\right)\right|^{2} \\
& \quad+\sum_{n=1}^{\infty} \frac{1}{\lambda_{n}^{2 \gamma}}\left|\int_{0}^{t}\left(F_{1}(u, v, \tau), \varphi_{n}\right)(t-\tau)^{\alpha-1} E_{\alpha, \alpha}\left(-\lambda_{n}(t-\tau)^{\alpha}\right) d \tau\right|^{2} \\
& =S_{3}(t)+S_{4}(t) .
\end{aligned}
$$

Since

$$
S_{3}(t) \leq C \sum_{n=1}^{\infty} \frac{1}{\lambda_{n}^{2 \gamma}}\left|\left(a_{1}, \varphi_{n}\right)\right|^{2} \leq C \frac{1}{\lambda_{1}^{2 \gamma}}\left\|a_{1}\right\|_{L^{2}}^{2}<\infty
$$

and

$$
\lim _{t \rightarrow 0}\left(E_{\alpha, 1}\left(-\lambda_{n} t^{\alpha}\right)-1\right)=0
$$

for each $n \in \mathbb{N}$, by using the Lebesgue dominated convergence theorem, we have

$$
\lim _{t \rightarrow 0} S_{3}(t)=0
$$

By Lemma 2, we have

$$
\begin{aligned}
S_{4}(t) & \leq C \sup _{0 \leq t \leq T}\left\|F_{1}\right\|_{L^{2}}^{2} \sum_{n=1}^{\infty} \frac{1}{\lambda_{n}^{2 \gamma}}\left|\int_{0}^{t} \tau^{\alpha-1} E_{\alpha, \alpha}\left(-\lambda_{n} \tau^{\alpha}\right) d \tau\right|^{2} \\
& \leq C \sup _{0 \leq t \leq T}\left\|F_{1}\right\|_{L^{2}}^{2} \sum_{n=1}^{\infty} \frac{1}{\lambda_{n}^{2 \gamma+2}}\left(1-E_{\alpha, 1}\left(-\lambda_{n} t^{\alpha}\right)\right)^{2} \\
& \leq C \sum_{n=1}^{\infty} \frac{1}{\lambda_{n}^{2 \gamma+2}}
\end{aligned}
$$


Since $\lambda_{n} \geq C n^{\frac{2}{d}}($ e.g. [21]), we obtain

$$
S_{4}(t) \leq C \sum_{n=1}^{\infty} \frac{1}{n^{\frac{4 \gamma+4}{d}}}<\infty
$$

for $\gamma>\frac{d}{4}-1$. Hence, the Lebesgue dominated convergence theorem yields

$$
\lim _{t \rightarrow 0} S_{4}(t)=0
$$

Thus we complete the proof of (ii).

Remark In the proof of Theorem 1, we have used the fact $\frac{1}{2} \leq \alpha<1$. But we do not know whether it still holds for the case $0<\alpha<\frac{1}{2}$. The energy estimation for the case $0<\alpha<\frac{1}{2}$ is technically more complicated and will be done in the future.

Theorem 2 Let $1<\alpha<2$, the initial data $\left(a_{1}, a_{2}\right) \in V$ and $\left.\partial_{t} u\right|_{t=0}=0,\left.\partial_{t} v\right|_{t=0}=0, x \in \Omega$.

Then there exists a unique solution $(u, v)$ to equations (1)-(4) with

$$
(u, v) \in C([0, T], V), \quad\left(\partial_{t}^{\alpha} u, \partial_{t}^{\alpha} v\right) \in C([0, T] ; H),
$$

and the following estimates hold

$$
\begin{aligned}
& \|u(\cdot, t)\|_{H^{2}}+\|v(\cdot, t)\|_{H^{2}} \leq C, \quad t \in[0, T], \\
& \left\|\partial_{t}^{\alpha} u(\cdot, t)\right\|_{L^{2}}+\left\|\partial_{t}^{\alpha} v(\cdot, t)\right\|_{L^{2}} \leq C, \quad t \in[0, T] .
\end{aligned}
$$

Proof By assumption (A.2) and equations (5)-(6), we have

$$
\begin{aligned}
\|u(\cdot, t)\|_{H^{2}}^{2} \leq & C\|L u(\cdot, t)\|_{L^{2}}^{2} \\
\leq & C \sum_{n=1}^{\infty} \lambda_{n}^{2}\left|\left(a_{1}, \varphi_{n}\right) E_{\alpha, 1}\left(-\lambda_{n} t^{\alpha}\right)\right|^{2} \\
& +C \sum_{n=1}^{\infty} \lambda_{n}^{2}\left|\left(\int_{0}^{t}\left(F_{1}(u, v, \tau), \varphi_{n}\right)(t-\tau)^{\alpha-1} E_{\alpha, \alpha}\left(-\lambda_{n}(t-\tau)^{\alpha}\right) d \tau\right)\right|^{2} \\
\leq & C\left\|a_{1}\right\|_{H^{2}}^{2}+C \int_{0}^{t}\left\|F_{1}(u, v)\right\|_{H^{2}}^{2} d \tau \\
\leq & C+C \int_{0}^{t}\left(\|u(\cdot, \tau)\|_{H^{2}}^{2}+\|v(\cdot, \tau)\|_{H^{2}}^{2}\right) d \tau .
\end{aligned}
$$

Similarly,

$$
\|v(\cdot, t)\|_{H^{2}}^{2} \leq C+C \int_{0}^{t}\left(\|u(\cdot, t)\|_{H^{2}}^{2}+\|v(\cdot, t)\|_{H^{2}}^{2}\right) d \tau .
$$

Combining the above two inequalities, and using Gronwall's inequality, we have

$$
\|u(\cdot, t)\|_{H^{2}}^{2}+\|v(\cdot, t)\|_{H^{2}}^{2} \leq C\left(\left\|a_{1}\right\|_{H^{2}},\left\|a_{2}\right\|_{H^{2}}, T, K_{3}, K_{4}, \alpha\right), \quad t \in[0, T] .
$$


Furthermore,

$$
\|u(\cdot, t)\|_{L^{\infty}\left(0, T ; H^{2}\right)} \leq C, \quad\|v(\cdot, t)\|_{L^{\infty}\left(0, T ; H^{2}\right)} \leq C .
$$

From equations (1)-(2), we can also get the estimate

$$
\left\|\partial_{t}^{\alpha} u(\cdot, t)\right\|_{L^{2}}^{2}+\left\|\partial_{t}^{\alpha} v(\cdot, t)\right\|_{L^{2}}^{2} \leq C .
$$

The proof of uniqueness is similar to the proof of Theorem 1 .

Corollary 1 Let $1<\alpha<2$ and the initial data $a_{1}=a_{2}=0$, then we have

$$
\|u(\cdot, t)\|_{\mathcal{D}\left((-L)^{\frac{\alpha-1}{\alpha}}\right)}+\|v(\cdot, t)\|_{\mathcal{D}\left((-L)^{\frac{\alpha-1}{\alpha}}\right)} \leq C, \quad t \in[0, T] .
$$

Proof From equation (5), we have

$$
\begin{aligned}
& \left\|(-L)^{\frac{\alpha-1}{\alpha}} u(\cdot, t)\right\|_{L^{2}}^{2} \\
& =\sum_{n=1}^{\infty} \lambda_{n}^{2 \frac{\alpha-1}{\alpha}}\left|\int_{0}^{t}\left(F_{1}(u, v, \tau), \varphi_{n}\right)(t-\tau)^{\alpha-1} E_{\alpha, \alpha}\left(-\lambda_{n}(t-\tau)^{\alpha}\right) d \tau\right|^{2} \\
& \quad \leq C \sum_{n=1}^{\infty} \sup _{0 \leq t \leq T}\left|\left(F_{1}(u, v, t), \varphi_{n}\right)\right|^{2}\left|\int_{0}^{t} \frac{\left(\lambda_{n} \tau^{\alpha}\right)^{\frac{\alpha-1}{\alpha}}}{1+\lambda_{n} \tau^{\alpha}} d \tau\right|^{2} .
\end{aligned}
$$

Since

$$
\sup _{\tau \geq 0} \frac{\left(\lambda_{n} \tau^{\alpha}\right)^{\frac{\alpha-1}{\alpha}}}{1+\lambda_{n} \tau^{\alpha}}=\frac{(\alpha-1)^{\frac{\alpha-1}{\alpha}}}{\alpha}
$$

we deduce that

$$
\left\|(-L)^{\frac{\alpha-1}{\alpha}} u(\cdot, t)\right\|_{L^{2}}^{2} \leq C T^{2}\left\|F_{1}(u, v)\right\|_{L^{\infty}\left(0, T ; L^{2}\right)}^{2} \leq C, \quad t \in[0, T] .
$$

Similarly,

$$
\left\|(-L)^{\frac{\alpha-1}{\alpha}} v(\cdot, t)\right\|_{L^{2}}^{2} \leq C, \quad t \in[0, T]
$$

Corollary 2 Let $1<\alpha<2$ and the initial data $a_{1}=a_{2}=0$, then we have

$$
\left\|\partial_{t} u(\cdot, t)\right\|_{\mathcal{D}\left((-L)^{-\gamma}\right)}+\left\|\partial_{t} v(\cdot, t)\right\|_{\mathcal{D}\left((-L)^{-\gamma}\right)} \leq C, \quad t \in[0, T],
$$

for $\gamma>\frac{d}{4}$.

Proof By Lemma 2, we have

$$
\partial_{t} u(\cdot, t)=\sum_{n=1}^{\infty}\left(\int_{0}^{t}\left(F_{1}(u, v, \tau), \varphi_{n}\right)(t-\tau)^{\alpha-2} E_{\alpha, \alpha-1}\left(-\lambda_{n}(t-\tau)^{\alpha}\right) d \tau\right) \varphi_{n}(x),
$$




$$
\partial_{t} \nu(\cdot, t)=\sum_{n=1}^{\infty}\left(\int_{0}^{t}\left(F_{2}(u, \nu, \tau), \varphi_{n}\right)(t-\tau)^{\alpha-2} E_{\alpha, \alpha-1}\left(-\lambda_{n}(t-\tau)^{\alpha}\right) d \tau\right) \varphi_{n}(x) .
$$

Therefore, by Lemma 1 and $\lambda_{n} \geq C n^{\frac{2}{d}}$, we have

$$
\begin{aligned}
& \left\|\partial_{t} u(\cdot, t)\right\|_{\mathcal{D}((-L)-\gamma)}^{2} \\
& \quad=\sum_{n=1}^{\infty} \frac{1}{\lambda_{n}^{2 \gamma}}\left|\int_{0}^{t}\left(F_{1}(u, v, \tau), \varphi_{n}\right)(t-\tau)^{\alpha-2} E_{\alpha, \alpha-1}\left(-\lambda_{n}(t-\tau)^{\alpha}\right) d \tau\right|^{2} \\
& \quad \leq \sum_{n=1}^{\infty} \frac{1}{\lambda_{n}^{2 \gamma}} \sup _{0 \leq t \leq T}\left|\left(F_{1}(u, v, t), \varphi_{n}\right)\right|^{2}\left|\int_{0}^{t} \tau^{\alpha-2} E_{\alpha, \alpha-1}\left(-\lambda_{n} \tau^{\alpha}\right) d \tau\right|^{2} \\
& \quad \leq C\left\|F_{1}(u, v)\right\|_{L^{\infty}\left(0, T ; L^{2}\right)}^{2} \sum_{n=1}^{\infty} \frac{1}{\lambda_{n}^{2 \gamma}} t^{2 \alpha-2} \\
& \quad \leq C\left\|F_{1}(u, v)\right\|_{L^{\infty}\left(0, T ; L^{2}\right)}^{2} T^{2 \alpha-2} \sum_{n=1}^{\infty} \frac{1}{n^{\frac{4 \gamma}{d}}}<\infty
\end{aligned}
$$

for $\gamma>\frac{d}{4}$. In the same way, we can also obtain the estimate

$$
\left\|\partial_{t} u(\cdot, t)\right\|_{\mathcal{D}\left((-L)^{-\gamma}\right)}^{2}<\infty
$$

Thus we complete the proof.

\section{Competing interests}

The authors declare that they have no competing interests.

\section{Authors' contributions}

The authors contributed equally to the writing of this paper. All authors read and approved the final manuscript.

\section{Acknowledgements}

Lang Li was partially supported by the NSFC under grant No. 11426069 and No. 61375006. Lingyu Jin was supported by the NSFC under grant No. 11101160 . Shaomei Fang was supported by the NSFC under grant No. 11271141. The authors would like to thank the anonymous referee for many helpful comments and suggestions.

Received: 2 October 2015 Accepted: 19 November 2015 Published online: 03 December 2015

\section{References}

1. Kilbas, AA, Srivastava, HM, Trujillo, JJ: Theory and Applications of Fractional Differential Equations. Elsevier, Amsterdam (2006)

2. Podlubny, I: Fractional Differential Equations. Academic Press, San Diego (1999)

3. Miller, KS, Ross, B: An Introduction to the Fractional Calculus and Fractional Differential Equations. Wiley, New York (1993)

4. Tarasov, V: Fractional Dynamics: Applications of Fractional Calculus to Dynamics of Particles, Fields and Media. Springer, Berlin (2010)

5. Nigmatullin, RR: The realization of the generalized transfer equation in a medium with fractal geometry. Phys. Status Solidi B 133, 425-430 (1986)

6. Metzler, R, Klafter, J: Boundary value problems for fractional diffusion equations. Physica A 278, 107-125 (2000)

7. Roman, HE, Alemany, PA: Continuous-time random walks and the fractional diffusion equation. J. Phys. A 27, 3407-3410 (1994)

8. Agarwal, OP: Solution for a fractional diffusion-wave equation defined in a bounded domain. Nonlinear Dyn. 29, 145-155 (2002)

9. Mainardi, F: The fundamental solutions for the fractional diffusion-wave equation. Appl. Math. Lett. 9, 23-28 (1996)

10. Eidelman, SD, Kochubei, AN: Cauchy problem for fractional diffusion equations. J. Differ. Equ. 199, 211-255 (2004)

11. Luchko, Y: Maximum principle for the general time-fractional diffusion equation. J. Math. Anal. Appl. 351, 218-223 (2009)

12. Luchko, Y: Initial boundary value problems for the generalized multi-term time-fractional diffusion equation. J. Math. Anal. Appl. 374, 538-548 (2011) 
13. Sakamoto, K, Yamamoto, M: Initial value/boundary value problems for fractional diffusion-wave equations and applications to some inverse problems. J. Math. Anal. Appl. 382, 426-447 (2011)

14. Gafiychuk, V, Datsko, B: Mathematical modeling of different types of instabilities in time fractional reaction-diffusion systems. Comput. Math. Appl. 59, 1101-1107 (2010)

15. Luchko, Y: Some uniqueness and existence results for the initial-boundary value problems for the generalized time-fractional diffusion equation. Comput. Math. Appl. 59, 1766-1772 (2010)

16. Bebernes, J, Bressan, A, Lacey, A: Total blow-up versus single-point blow-up. J. Differ. Equ. 73, 30-44 (1988)

17. Datsko, B, Luchko, Y: Complex oscillations and limit cycles in autonomous two-component incommensurate fractional dynamical systems. Math. Balk. 26, 65-78 (2012)

18. Datsko, B, Luchko, Y, Gafiychuk, V: Pattern formation in fractional reaction-diffusion systems with multiple homogeneous states. Int. J. Bifurc. Chaos 22, 1250087 (2012)

19. Liu, W: Well-posedness of stochastic partial differential equations with Lyapunov condition. J. Differ. Equ. 255, 572-592 (2013)

20. Miller, KS, Samko, SG: Completely monotonic functions. Integral Transforms Spec. Funct. 12, 389-402 (2001)

21. Courant, R, Hilbert, D: Methods of Mathematical Physics, vol. 1. Interscience, New York (1953)

\section{Submit your manuscript to a SpringerOpen ${ }^{\circ}$ journal and benefit from:}

- Convenient online submission

Rigorous peer review

- Immediate publication on acceptance

- Open access: articles freely available online

- High visibility within the field

- Retaining the copyright to your article 